Research article

\title{
Tau mRNA 3'UTR-to-CDS ratio is increased in Alzheimer disease
}

\author{
Vega García-Escudero a,b,1, Ricardo Gargini a , Patricia Martín-Maestro ${ }^{\mathrm{a}, \mathrm{b}, 2}$, \\ Esther García ${ }^{\mathrm{a}, \mathrm{b}}$, Ramón García-Escudero ${ }^{\mathrm{c}, \mathrm{d}, \mathrm{e}}$, Jesús Avila ${ }^{\mathrm{a}, \mathrm{b}, *}$ \\ a Centro de Biología Molecular Severo Ochoa (UAM-CSIC), Nicolás Cabrera 1, Cantoblanco, 28049 Madrid, Spain \\ ${ }^{\mathrm{b}}$ Centro de Investigación Biomédica en Red de Enfermedades Neurodegenerativas (CIBERNED), Valderrebollo 5, 28031 Madrid, Spain \\ ${ }^{\mathrm{c}}$ Molecular Oncology Unit, CIEMAT, Complutense 40, 28040 Madrid, Spain \\ d Biomedical Research Institute I+12, University Hospital 12 de Octubre, Avenida de Córdoba s/n, 28041 Madrid, Spain \\ e Centro de Investigación Biomédica en Red de Cáncer (CIBERONC), Monforte de Lemos 3-5, 28029 Madrid, Spain
}

\section{H I G H L I G H T S}

- Healthy mouse and human brain show a balanced 3'UTR-to-CDS MAPT ratio.

- MAPT mRNA is decreased in Alzheimer disease, whereas tau protein is increased.

- Neuronal markers are low in Alzheimer brain, in contrast to glial cell markers.

- Alzheimer patient brains have an increased 3'UTR-to-CDS MAPT ratio.

\section{A R T I C L E I N F O}

\section{Article history:}

Received 8 May 2017

Received in revised form 1 July 2017

Accepted 5 July 2017

Available online 8 July 2017

\section{Keywords:}

Tau (MAPT)

$3^{\prime}$ UTR

CDS

mRNA

Alzheimer disease

\begin{abstract}
A B S T R A C T
Neurons frequently show an imbalance in expression of the $3^{\prime}$ untranslated region ( $3^{\prime} \mathrm{UTR}$ ) relative to the coding DNA sequence (CDS) region of mature messenger RNAs (mRNA). The ratio varies among different cells or parts of the brain. The Map2 protein levels per cell depend on the $3^{\prime} U T R$-to-CDS ratio rather than the total mRNA amount, which suggests powerful regulation of protein expression by $3^{\prime}$ UTR sequences. Here we found that MAPT (the microtubule-associated protein tau gene) $3^{\prime}$ UTR levels are particularly high with respect to other genes; indeed, the 3'UTR-to-CDS ratio of MAPT is balanced in healthy brain in mouse and human. The tau protein accumulates in Alzheimer diseased brain. We nonetheless observed that the levels of RNA encoding MAPT/tau were diminished in these patients' brains. To explain this apparently contradictory result, we studied MAPT mRNA stoichiometry in coding and non-coding regions, and found that the 3'UTR-to-CDS ratio was higher in the hippocampus of Alzheimer disease patients, with higher tau protein but lower total mRNA levels. Our data indicate that changes in the 3'UTR-to-CDS ratio have a regulatory role in the disease. Future research should thus consider not only mRNA levels, but also the ratios between coding and non-coding regions.
\end{abstract}

(C) 2017 Elsevier B.V. All rights reserved.

\section{Introduction}

Tau, a microtubule-associated protein encoded by the MAPT gene, is involved in multiple neuropathologies. Tau is expressed

Abbreviations: MAPT, Microtubule associated protein tau; UTR, untranslated region; CDS, coding DNA sequence; mRNA, messenger RNA; mTOR, mammalian target of rapamycin; 5'TOP, 5' terminal oligopyrimidine; RIN, RNA integrity number.

* Corresponding author at: Centro de Biología Molecular Severo Ochoa, Nicolás Cabrera 1, Cantoblanco, 28049 Madrid, Spain.

E-mail addresses: javila@cbm.csic.es, jesus.avila@csic.es (J. Avila).

1 Present address: Departamento de Anatomía, Histología y Neurociencia, Facultad de Medicina UAM, Arzobispo Morcillo 4, 28029 Madrid, Spain.

2 Present address: Brain and Mind Research Institute, Weill Cornell Medical College, Cornell University,407 E 61 st St., New York, NY 10065, USA. in brain, specifically in neuronal cells. In the adult human brain, the MAPT gene is composed of 16 exons on chromosome $17 \mathrm{q} 21$ that give rise to six tau isoforms, which are generated by alternative splicing of exons 2,3 and $10[1,2]$. The MAPT gene is transcribed into its nuclear RNA which, after intron splicing and polyadenylation, becomes MAPT messenger RNA (mRNA) that bears its $5^{\prime}$ UTR (untranscribed region), the coding exons (CDS) and its $3^{\prime}$ UTR region; this transcription unit is a single entity that will be translated into tau protein.

In its normal physiological function, tau is differentially expressed during neuron development [3]. In young neurons, tau is distributed equally between cell body and neurites. When the axon emerges and the neuron is polarized, tau becomes enriched in the axon and to a lesser extent, in dendrites and the nucleus. The process that underlies polarized distribution in mature neu- 
rons remains poorly understood, as it takes place at both mRNA and protein levels [3]. MAPT mRNA is distributed throughout the proximal axon due to an axon localization signal in the $3^{\prime}$ UTR region [4]. Tau is also preferentially translated in axons, which is dependent on mTOR (mammalian target of rapamycin) signaling [5]. The MAPT $5^{\prime}$ UTR has a $5^{\prime}$ terminal oligopyrimidine (5'TOP) track that mediates mTOR-governed protein synthesis [6]. Differential distribution of tau and its isoforms allows diverse functions [7]. The MAPT 3'UTR binds several proteins, such as HuD, IMP-1, G3BP, Ilf3, and NF90, that are potentially associated to MAPT mRNA axonal localization $[8,9]$. Perturbation of MAPT mRNA sorting and distribution might induce toxic gain of function and contribute to taupathies [10].

MAPT 3'UTR also has important cis-elements that decrease its RNA expression [7], as well as several micro-RNA binding sites such as miR219, miR34a, miR35c-5p, miR485-5p and miR132-3p; this last is strongly downregulated in Alzheimer [11-15].

In this study, we show that several genes are unbalanced in Alzheimer pathology. This misregulation involves notable loss of neuronal markers, in contrast to increased glial hallmarks and unaffected microglial and oligodendroglial markers. We observed that the neuronal marker MAPT mRNA is reduced, whereas tau protein is increased. In addition, the ratio of $3^{\prime} U T R$ to the CDS, which appears to be balanced in human and mouse normal brain tissue, was significantly increased in Alzheimer disease. Our data indicate that the amount of tau protein does not correlate with the mRNA levels, and highlight the importance of non-coding regions in the regulation of protein synthesis.

\section{Materials and methods}

\subsection{RNA-seq data analysis}

RNA-seq data from mouse and human brain samples were downloaded from the NCBI Sequence Read Archive (SRA) repository using the SRP053398 and SRP004879 identifiers, respectively. SRP053398 contains 8 mRNA samples from brain striatum of WT q175 mice [3] that were analyzed with Illumina HiSeq 2000, and paired-end reads of 50 bases. SRP004879 contains 3 pooled mRNA samples from normal human brain (whole brain, frontal lobe, and temporal lobe) and 3 mRNA samples from Alzheimer patients (whole brain, frontal lobe, and temporal lobe; [16]) (see Table 1), analyzed with Illumina Genome Analyzer II, with singleend reads of 36 bases. Raw data were downloaded as SRA files, converted to FASTQ files using the NCBI SRA-Toolkit (v. 2.6.2), and mapped to genomes using TopHat2 (v. 2.1.1) [17]. We used transcript annotations files gencode.v24.annotation.gtf (human) and gencode.vM4.annotation.gtf (mouse) (both obtained from the GENCODE website, http://www.gencodegenes.org). Genome versions GRCh38.77 (human) and GRCm38.76 (mouse) were used, obtained from the ENSEMBL website (http://www.ensembl.org/index.html). Read depth from specific exons and 3'UTR regions were obtained directly using the output BAM files from TopHat2 using SAMtools (v. 1.3.1) [18]. Briefly, read depth was calculated for the last three exons and the $3^{\prime}$ UTR region for human and mouse samples. CDS expression was calculated as the average read depth of the three exons, thus ruling out differential coverage between $5^{\prime}$ and $3^{\prime}$ regions within mRNAs due to retrotranscription using oligo-dT before massive sequencing.

\subsection{Brain tissue}

Hippocampal brain samples from sporadic Alzheimer disease patients and control subjects were obtained from the Banco de Tejidos (Fundación CIEN, Instituto de Salud Carlos III, Madrid, Spain). Based on quantitative pathological features, the Alzheimer brain specimens $(n=16)$ were classified according Braak stages II $(n=3)$, III $(n=2), \mathrm{V}(n=7)$ and VI $(n=4)$, and non-demented control subjects $(n=8)$ (see Supplementary Table S1 in the online version at DOI: 10.1016/j.neulet.2017.07.007 for further subject information). Written informed consent was obtained from all patients.

\subsection{Western blot analyses}

Frozen brain tissue was homogenized using a TissueLyser (Retsch MM300, Qiagen, Hilden, Germany) (30 Hz, $5 \mathrm{~min}$ ) with 5-mm stainless steel beads (69989, Qiagen) in $50 \mathrm{mM}$ Tris- $\mathrm{HCl}$ $\mathrm{pH} 7.5,300 \mathrm{mM} \mathrm{NaCl}, 0.5 \%$ SDS (sodium dodecyl sulphate) and $1 \%$ Triton $\mathrm{X}-100$. The homogenate was incubated $\left(15 \mathrm{~min}, 95^{\circ} \mathrm{C}\right)$, centrifuged $(16,100 \times g, 10 \mathrm{~min})$ and supernatant considered brain extract. Protein concentration was measured using the Dc protein assay kit (500-0111, Bio-Rad). From each sample, $30 \mu \mathrm{g}$ total protein were resolved on a $10 \%$ Bis-Tris gel and transferred to PVDF membranes. Blots were probed with an anti-tau antibody (antibody 7.51) [19], followed by horseradish peroxidase-conjugated anti-mouse antibody (Dako, Glostrup, Denmark). Tau expression was quantified by measuring the ECL signal per lane with ImageJ software (http://rsbweb.nih.gov/ij/).

\subsection{Microarray analysis}

The expression profiling by array (Affymetrix Human Gene 1.0 ST Array) was retrieved from the Hisayama study (GEO id: 4758) [20] for following genes in human brain samples classified as healthy $(n=47)$ vs. Alzheimer disease $(n=32)$ : MAPT, MAP2, TUBB3, NeuN, Syt1, Graba1 and Snap25 as neuronal markers; GFAP, S100B, Adhl1l1 and Aqp4 as astrocytic markers; IBA1, CD68, CD45 and CD11b as microglial markers; GalC, Mog, SOX10 and CX47 as oligodendrocytic markers [21]. Differences in expression of each gene between healthy and Alzheimer samples were calculated using Student's t-test.

\subsection{Quantitative real-time PCR assays}

Frozen brain tissue was homogenized using a TissueLyser in conditions as above, in $700 \mu \mathrm{l}$ QIAzol Lysis Reagent (79306, Qiagen). RNA was extracted using miRNeasy Mini Kit (217004, Qiagen) with RNase-Free DNase Set (79254, Qiagen) following manufacturer's guidelines. The RNA integrity number (RIN) of each sample was calculated using the Agilent 2100 Bioanalyzer system (Agilent Technologies), and only RNAs with RIN $>5$ were used for RT-qPCR. RNA was retrotranscribed with the Transcriptor First Strand cDNA Synthesis Kit (04379012001, Roche) using $20 \mathrm{ng} / \mu \mathrm{l}$ RNA with random primers. Quantitative PCR was performed in a LightCycler480 (Roche) in the following conditions: $50^{\circ} \mathrm{C}$ for $2 \mathrm{~min}, 95^{\circ} \mathrm{C}$ for $10 \mathrm{~min}$, and 40 cycles of $95^{\circ} \mathrm{C}$ for $15 \mathrm{~s}$ and $60^{\circ} \mathrm{C}$ for $1 \mathrm{~min}$. Oligonucleotides used for detection of MAPT-CDS: MAPT-E11-E13-fw GTCGAAGATTGG GTCCCTGG and MAPT-E11-E13-rv GACACCACTGGCGACTTGTA, for MAPT-3'UTR: MAPT-3UTR-B-fw TTTTGGCCACTTTGCAGACC and MAPT-3UTR-B-rV TCTCCCAA GAGGCACAAGTC, and for TUBB3: TUBB3-fw AACGAGGCCTCTTCTCACAA and TUBB3-rv GGCCTGAAGAGATGTCCAAA. Gene expression was normalized to GAPDH expression using TaqMan primer human GAPDH (Hs02758991_g1, Applied Biosystems).

\subsection{Statistical analysis}

Quantitative data, represented as mean \pm SD or SEM were compared between groups using the two-tailed Student's $t$-test. 
Table 1

Source of total RNA from brain tissue samples.

\begin{tabular}{|c|c|c|c|c|}
\hline condition & sample & gender & Age (years) & Source \\
\hline \multirow[t]{3}{*}{ normal } & Whole brain & 13 male; 10 female & $23-86$ & Ambion \\
\hline & Frontal lobe & 5 male & $22-29$ & Capital Biosciences \\
\hline & Temporal lobe & 5 male & $23-29$ & Capital Biosciences \\
\hline \multirow[t]{3}{*}{ Alzheimer's disease } & Whole brain & 1 male & 87 & Capital Biosciences \\
\hline & Frontal lobe & 1 male & 87 & Capital Biosciences \\
\hline & Temporal lobe & 1 male & 80 & Capital Biosciences \\
\hline
\end{tabular}

http://journals.plos.org/plosone/article?id=10.1371/journal.pone.0016266.

Differences are presented with $P$ value $\left({ }^{*} p \leq 0.05 ;{ }^{* *} p \leq 0.01 ;{ }^{* * *}\right.$ $p \leq 0.001$; N.S. not significant).

\section{Results}

\subsection{MAPT $m R N A 3^{\prime} U T R-t o-C D S$ ratio is balanced in human and mouse brain}

Map2 has relatively similar 3'UTR-to-CDS expression in dopaminergic neurons as seen in RNA-seq analysis; some mouse brain regions nonetheless show unbalanced 3'UTR-to-CDS expression [22]. Cells with a 3' UTR-to-CDS ratio >3 do not express Map2 [22]. We studied this ratio for MAPT in published RNA-seq samples from mouse brain striatum [23], and found a balanced 3'UTR-to-CDS ratio in the eight wild-type mice (Fig. $1 \mathrm{~A}$ and $\mathrm{B}$ ). In a similar assay using reported human brain RNA-seq data [16] from whole brain and temporal and frontal lobes of healthy individuals (Fig. 1C, see Table 1), we found moderate imbalance, with slightly higher amounts of $3^{\prime}$ UTR compared to CDS in all samples (Fig. 1D).

\subsection{MAPT mRNA is reduced in Alzheimer disease patients}

Tau protein accumulates in Alzheimer disease brain [24], and advance of the pathology correlates with this accumulation [25]. Our collection of hippocampal brain samples showed a strong increase in tau protein levels in Alzheimer patients $(n=16)$ vs. non-demented controls $(n=8)$ (Fig. 2A, Supplementary Table S1 in the online version at DOI: $10.1016 /$ j.neulet.2017.07.007). Microarray data from control $(n=47)$ and Alzheimer disease brain samples $(n=32)$ from the Hisayama study [20] nonetheless showed significantly lower MAPT mRNA levels in Alzheimer vs. non-demented brain (Fig. 2B, Table 2). Given this apparent contradiction, we hypothesized that the proportion of neurons in Alzheimer brains was reduced as a result of neuronal degeneration.

To test this hypothesis, we analyzed several neuronal markers [26] in this sample set and found that neuronal structural genes such as MAP2 or TUBB3 ( $\beta$ III-tubulin), nuclear protein genes such as NeuN, and other neuronal markers were significantly lower in Alzheimer vs. control brain (Fig. 2B, Table 2). Analysis of markers for other cell components of the brain indicated that all astrocyte-associated markers were increased in Alzheimer brain (Fig. 2C), whereas those for microglia (Fig. 2D) and oligodendrocytes (Fig. 2E) showed no marked differences (Table 2). We thus identified a clear imbalance between tau protein and mRNA levels in Alzheimer patient brains.

\subsection{MAPT RNA 3'UTR-to-CDS ratio is increased in Alzheimer disease}

Although MAPT 3'UTR was only moderately enriched in human brain samples, we sought to determine its functional relevance. Given the importance of tau in Alzheimer pathology, we studied the 3'UTR-to-CDS ratio in patient brain mRNA samples (Fig. 3A, Table 1). Differences between MAPT 3'UTR and CDS were increased in Alzheimer samples, in which the number of 3'UTR reads doubled those of the CDS region, with a $\geq 2$-fold increase (Fig. 3B). We validated this result in a RT-qPCR assay in hippocampal brain samples from non-demented controls $(n=8)$ and Alzheimer patients ( $n=16$ ) (Supplementary Table S1 in the online version at DOI: 10. 1016/j.neulet.2017.07.007), using random primers for retrotranscription rather than oligo-dT, to avoid enrichment in $3^{\prime}$ poly-A regions. Results showed significant differences in $3^{\prime}$ UTR levels between non-demented and Alzheimer samples (Fig. 3C). There was a slight decrease in MAPT CDS levels in Alzheimer brain samples, in which we found high tau levels (Fig. 1A). This reduction might be related to the decreased neuron numbers and increased gliosis in these patients [27]; indeed, mRNA levels for the neuronal marker TUBB3 was significantly reduced in Alzheimer samples (Fig. 3C). To confirm this idea, we studied the ratio of MAPT CDS and 3'UTR normalized to neuron number as estimated by TUBB3 expression. Although MAPT CDS was similar in non-demented and Alzheimer patients (Fig. 3D and E), the MAPT 3'UTR was greatly increased in Alzheimer patient brains when normalized to neuron number (Fig. 3D). In addition, the MAPT 3'UTR-to-CDS ratio in these samples was significantly increased in Alzheimer relative to non-demented control brain (Fig. 3F).

\section{Discussion}

Studies in neurons show an unbalanced 3'UTR-to-CDS ratio for some mRNAs. As this imbalance might correlate with variations in protein expression involving several neuronal markers including some MAP [22], we sought to characterize MAPT mRNA stoichiometry, focusing on 3'UTR abundance relative to CDS. We hypothesized that variations in Alzheimer patient MAPT 3'UTR correlate with changes in tau protein levels, which would offer an additional mechanism to explain the tau pathology associated with the disease.

Physiological and pathological expression of the MAPT/tau gene shows complex regulation of both mRNA expression and localization [7]. We demonstrated reduced MAPT mRNA expression in Alzheimer brain, which we explain as a result of neuron loss in these patients, as MAPT levels relative to the constitutive neuronal marker TUBB3 were unchanged in healthy and diseased tissue. An earlier study reported increased MAPT mRNA levels in hippocampus of Alzheimer disease and other dementia patients [28]. Here we report that quantitative PCR, microarray and RNA-seq analyses indicated reduced MAPT mRNA levels. The difference between the two studies could be due to distinct experimental approaches. The work by Barton et al. used in situ hybridization to detect MAPT RNA, with a probe located at the beginning of the CDS and normalized to total poly-A RNA [28]. In our qPCR analysis, RNA integrity was confirmed for each sample; in addition, we used intron-spanning oligonucleotides that rule out genomic DNA contamination and are directed against the end of the CDS and the beginning of the 3'UTR. Our data were normalized to GAPDH mRNA, which was tested for stability in our sample set. In addition, we validated our 
A

Mouse Mapt
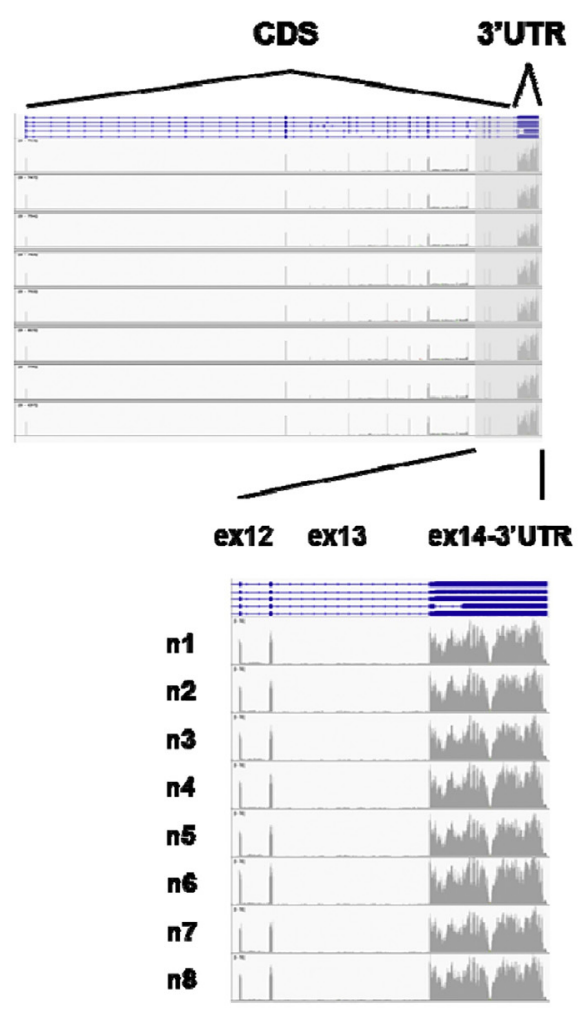

$\mathrm{B}$

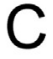

Human MAPT

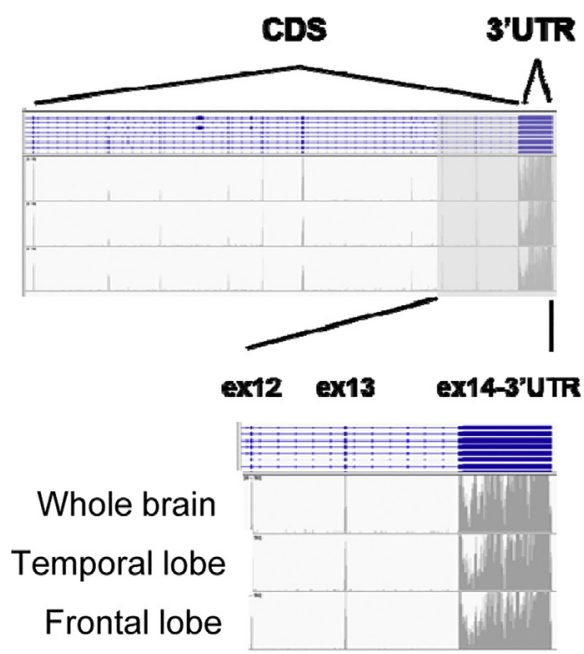

$\mathrm{D}$
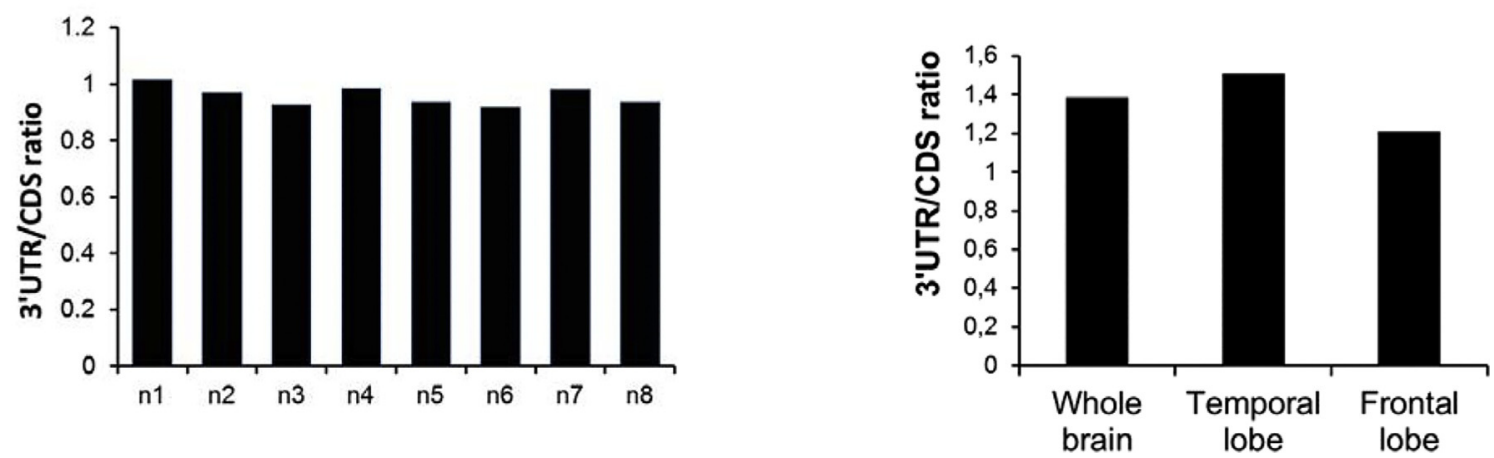

Fig. 1. RNA-seq analysis shows a balanced MAPT 3'UTR-to-CDS ratio.

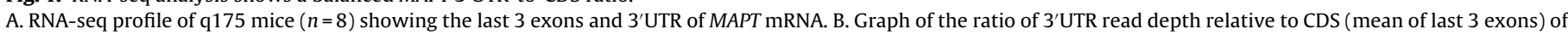

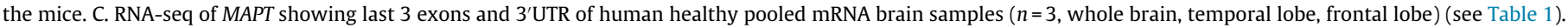
D. MAPT 3'UTR-to-CDS ratio of samples in C.

qPCR results by microarray and RNA-seq analysis, both accurate quantitative techniques.

On the other hand, whole-exome sequencing of 522 early-onset cases and 584 controls showed that $<1 \%$ of Alzheimer patients (4/522) had microduplications in chromosome 17 at the MAPT locus [29]. This analysis showed that this rare copy number variation in Alzheimer patients, which correlated with significantly increased MAPT mRNA levels in their blood cells. It could be of interest to explore MAPT mRNA levels in the brains of patients that harbor this duplication. The fact that an increased MAPT mRNA level correlated with Alzheimer pathology in a small percentage of cases contrasts with our finding of decreased mRNA levels, and indicates that an RNA stoichiometric imbalance could have a fundamental role in the disease. Further studies are needed to fully understand the involvement of RNA levels in this pathology.

We observed an inverse correlation between MAPT mRNA and tau levels. Another report described correlation between tau and MAPT mRNA levels during development [30]. However, MAPT mRNA can be transcribed in cells several days before tau protein is actively translated [31], which suggests that factors other than mRNA levels might determine activation of translation to protein. High tau levels when mRNA is decreased might indicate a protein degradation defect. The principal tau degradation pathways are ubiquitin-proteasome and autophagy-lysosome systems [13]. 
A
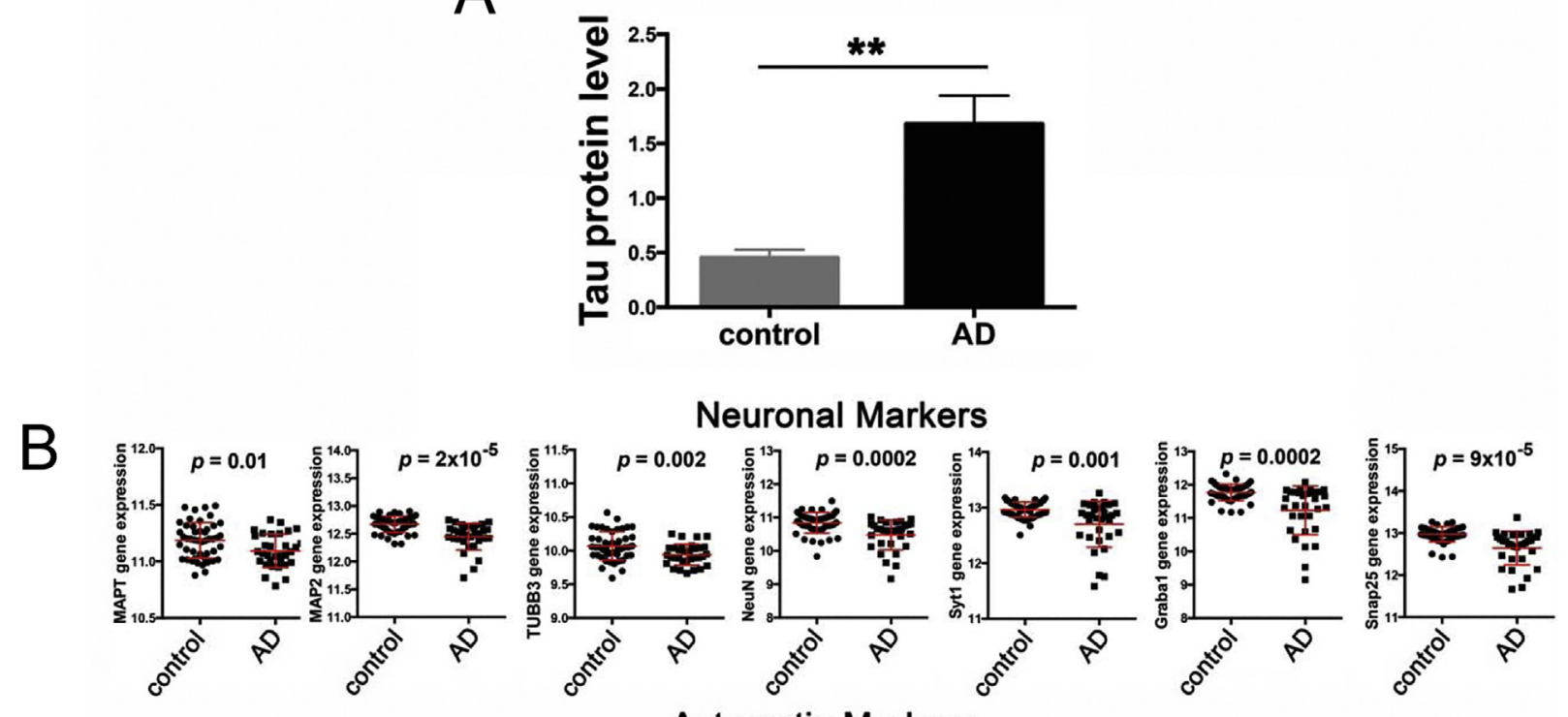

Astrocytic Markers

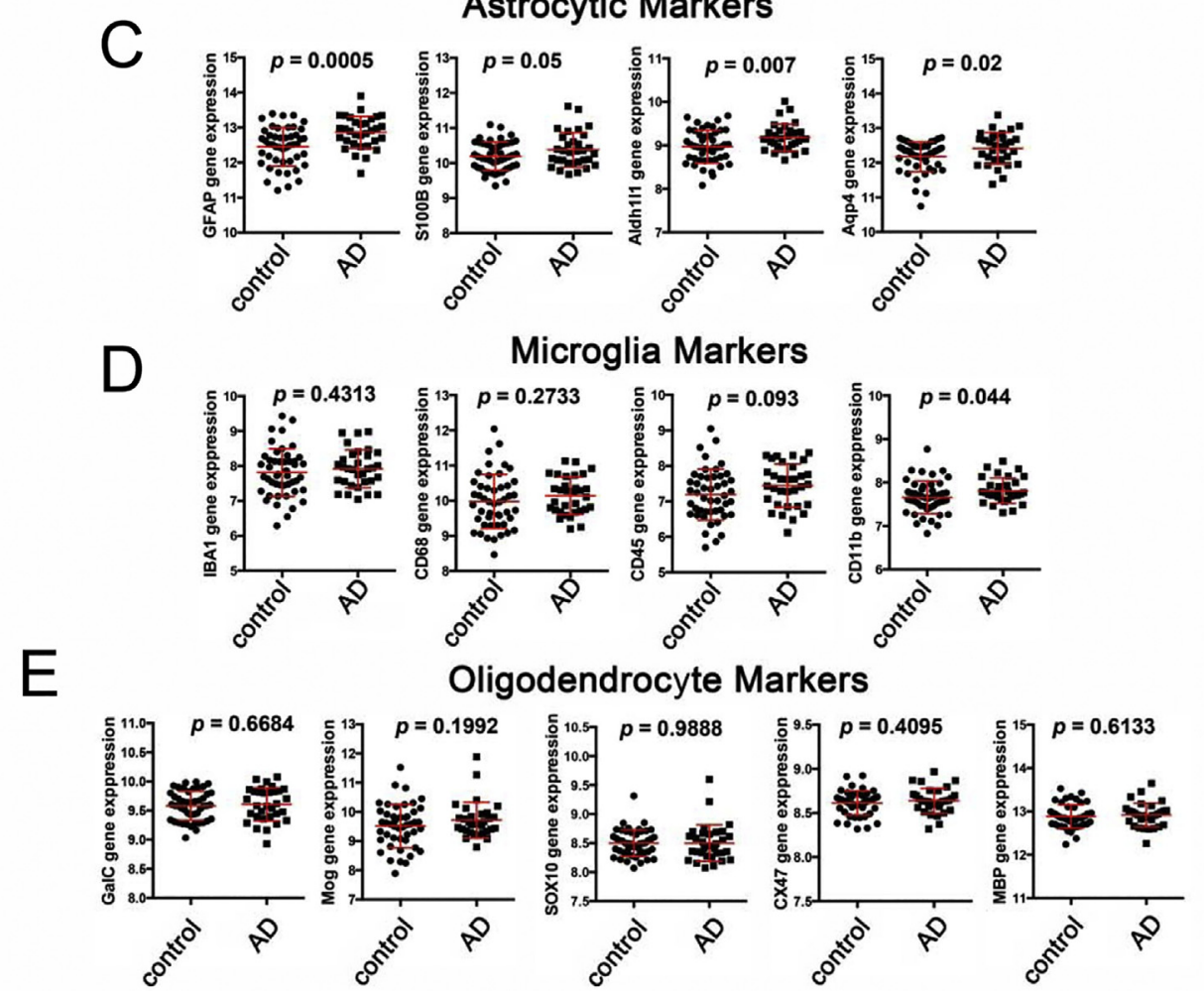

Fig. 2. Unbalanced expression of tau and neuronal markers in Alzheimer brain.

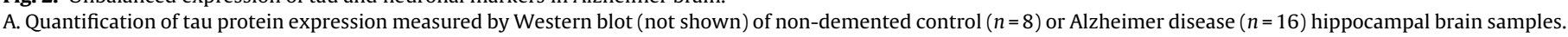

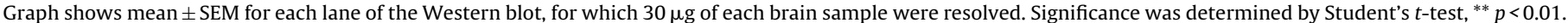

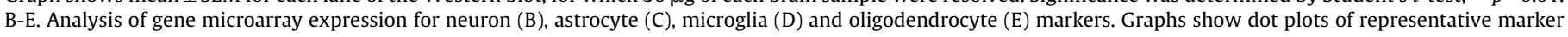

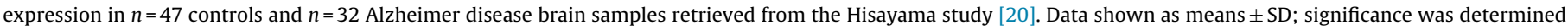
by Student's $t$-test and is shown for each graph.

Proteasome degradation is diminished in Alzheimer disease patient brain [32], and posttranslational modifications of tau such as phosphorylation or aggregation might inhibit proteasome degradation [33]. Alterations in the autophagy pathway have been demonstrated in the disease [34]; tau accumulation might thus be a result of deficient degradation rather than an increase in its synthesis. Moreover, mTORC1 signaling is increased in Alzheimer brain [11] and inhibits autophagy [35], thus contributing to tau accumulation. mTORC1 activation induces MAPT mRNA translation into protein by recognition of the 5'TOP motif in its 5'UTR [6], another possible mechanism of tau protein increase. Finally, not only tau levels but also its posttranslational modifications, including phosphorylation, glycosylation, glycation, prolyl-isomerization, cleavage or truncation, nitration, polyamination, ubiquitination, sumoylation, oxidation and aggregation, might have a fundamental role in Alzheimer pathogenesis [36]. Further study is needed to determine how MAPT 3'UTR levels correlate with these modifications in addition to the protein increase. 


\section{A Human MAPT}

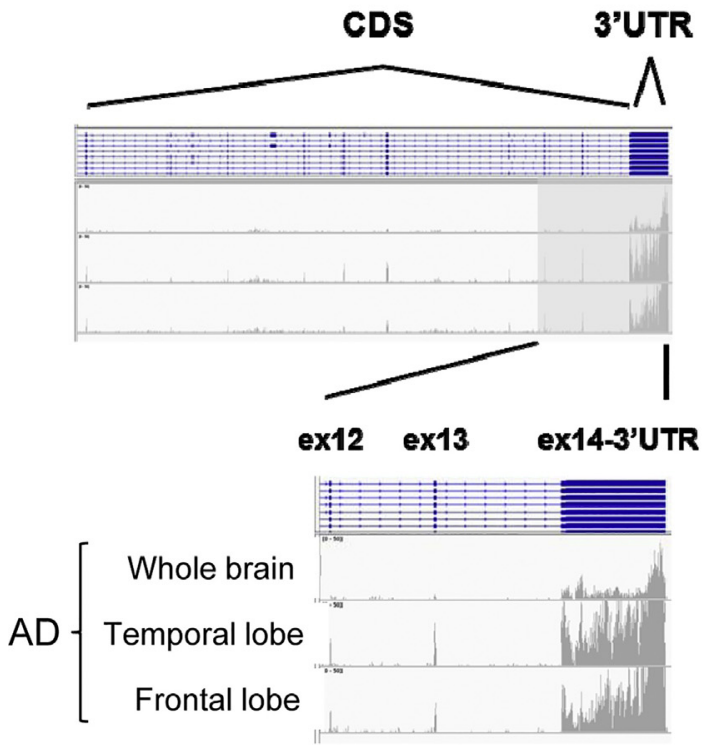

B

C
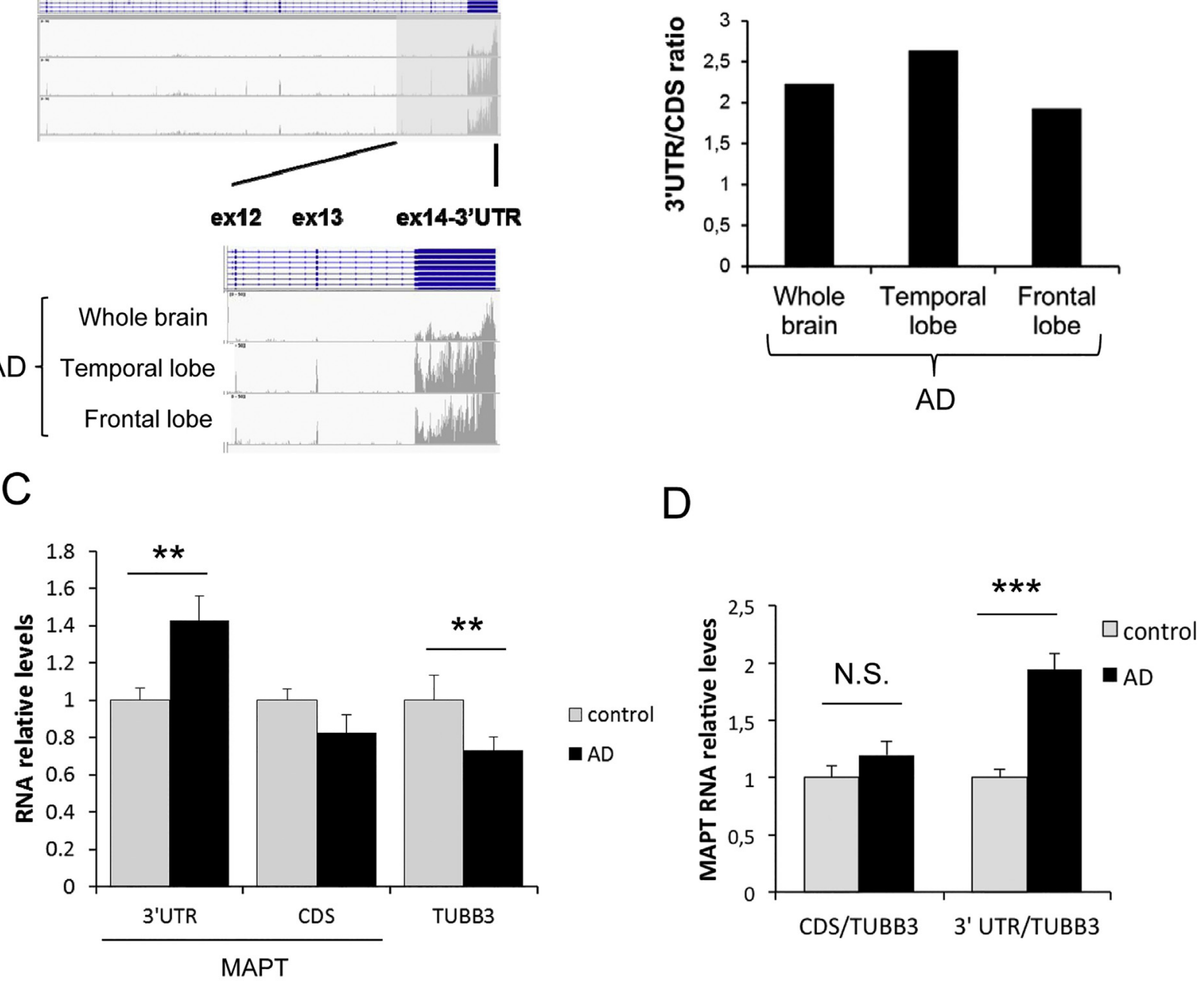

E

F
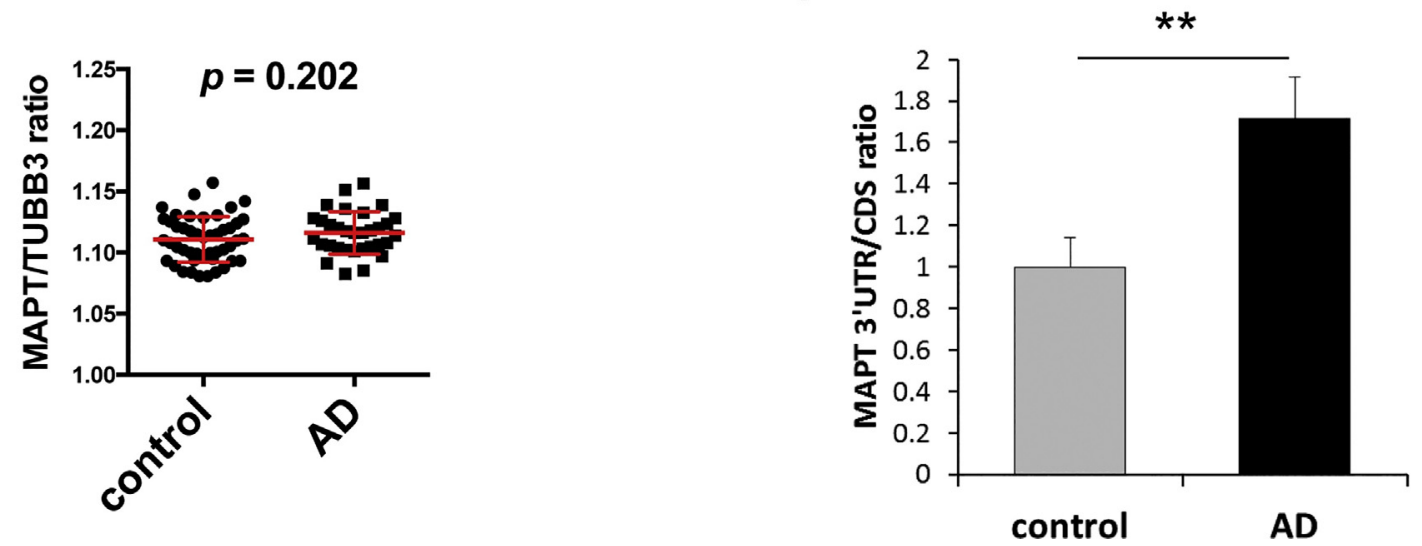

Fig. 3. MAPT $3^{\prime} U T R-$ to-CDS ratio is increased in Alzheimer disease.

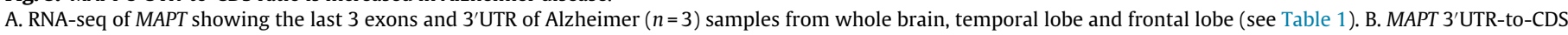

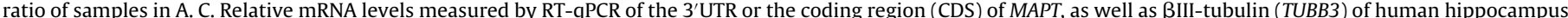

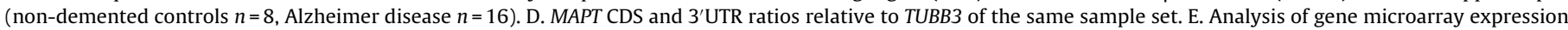

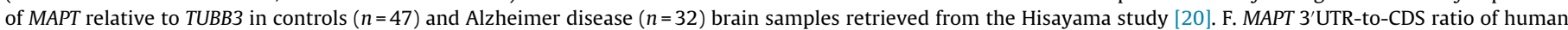

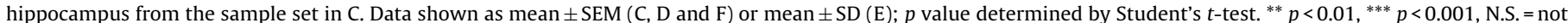
significant. 
Table 2

Changes in expression levels of markers in Alzheimer brain using gene microarray.

\begin{tabular}{|c|c|c|c|c|c|c|c|}
\hline \multirow[b]{2}{*}{ GENE } & \multicolumn{7}{|c|}{ Neuronal markers } \\
\hline & MAPT & MAP2 & TUBB3 & NeuN & Syt1 & Graba1 & Snap25 \\
\hline
\end{tabular}

\begin{tabular}{|c|c|c|c|c|}
\hline \multirow[b]{2}{*}{ Gene } & \multicolumn{4}{|c|}{ Astrocytic markers } \\
\hline & GFAP & S100B & Aldh1l1 & Aqp4 \\
\hline
\end{tabular}

\begin{tabular}{|c|c|c|c|c|}
\hline \multirow[b]{2}{*}{ Gene } & \multicolumn{4}{|c|}{ Microglial markers } \\
\hline & IBA1 & CD68 & CD45 & CD11b \\
\hline AD vs. control & N.S. & N.S. & N.S. & \\
\hline
\end{tabular}

\begin{tabular}{|c|c|c|c|c|c|}
\hline \multirow[b]{2}{*}{ Gene } & \multicolumn{5}{|c|}{ Oligodendrocyte markers } \\
\hline & GalC & Mog & SOX10 & CX47 & $\overline{\mathrm{MBP}}$ \\
\hline AD vs. control & N.S. & N.S. & N.S. & N.S. & N.S. \\
\hline
\end{tabular}

The 5'UTR region is involved in ribosome recruitment to mRNA and start codon choice [37]; less is known about the 3'UTR region. The MAPT 3'UTR region was characterized and was implicated in MAPT mRNA stabilization in neuronal cells [4,38]. This region contains AU-rich elements (ARES) that could direct poly-A tail addition to the end of MAPT mRNA.

Different amounts are reported of coding region (exons) and 3'UTR sequences in several neuronal genes [22]. Some genes have a 3'UTR without a CDS [39], although it remains to be clarified whether this results from expression of a RNA from $3^{\prime} \mathrm{UTR}$, or to distinct degradation of CDS and 3'UTR regions. In addition, a high 3'UTR-to-CDS ratio can lead to a decrease in protein levels [22].

We observed a balanced 3'UTR-to-CDS MAPT ratio in normal mouse and human tissue, similar to that described for Map2 [22]. In individual cells, however, these authors found that a high 3'UTR-to-CDS ratio coincided with reduced protein expression. We found an increased MAPT mRNA 3'UTR-to-CDS ratio in Alzheimer that coincides with increased tau levels, in contrasts those findings for Map2. A question that arises from these data is whether the increased 3'UTR-to-CDS MAPT ratio promotes mTORC1-dependent translation and thus allows the aberrant tau expression observed in Alzheimer brain. This question must be explored, taking into consideration the complexity of MAPT $3^{\prime}$ UTR, not only due to its different-length isoforms but also to the many proteins that bind this mRNA region.

\section{Conclusions}

Here we describe an inverse correlation in MAPT mRNA and tau protein levels, and an increased 3'UTR-to-CDS ratio in Alzheimer patient brain compared to non-demented controls. Reduction of the tau levels could be beneficial for disorders like Alzheimer, in which intracellular tau accumulation could be toxic to neurons [26,40-42], and induce synaptic impairment and memory deficits in mice $[43,44]$. Understanding the regulation of tau translation and the potential involvement of non-coding RNA regions could be a clue for treatment of such disorders. Our results suggest that disease models in which complete mRNA is used, including $5^{\prime}$ and $3^{\prime} \mathrm{UTR}$, are most appropriate for the study of Alzheimer disease, as they might be key regulatory elements in tau protein expression.

\section{Conflict of interest}

All authors declare that they have no conflict of interest.

\section{Author contributions}

G.E, R.G, P.M.M, E.G. and R.G.E performed the experiments. V.G.E, R.G. and R.G.E. designed the experiments and analyzed the data. V.G.E, R.G., R.G.E and J.A. wrote the manuscript, and provided editorial suggestions and criticisms.

\section{Acknowledgements}

We thank Dr. Alberto Rábano for human brain samples (Fundación CIEN, Instituto de Salud Carlos III, Madrid, Spain) and Dr. Marta Dueñas (CIEMAT, Madrid) for help in RNA extraction from brain samples. We are grateful to Silvia N. Vázquez (Genomics Unit, Fundación Parque Científico de Madrid) for advice and technical aid in qPCR analysis, and to Catherine Mark for editorial assistance.

This work was supported by the Spanish Ministerio de Economía y Competitividad (SAF 2011 program, project 24841 and grant BES-2012-055068) and the Centro de Investigación en Red de Enfermedades Neurodegenerativas (CIBERNED, ISCIII, JA). The funders had no role in study design, data collection and analysis, decision to publish, or preparation of the manuscript.

\section{References}

[1] A. Andreadis, Tau splicing and the intricacies of dementia, J. Cell. Physiol. 227 (2012) 1220-1225

[2] T. Guo, W. Noble, D.P. Hanger, Roles of tau protein in health and disease, Acta Neuropathol. (Berl.) 133 (2017) 665-704.

[3] P. Langfelder, J.P. Cantle, D. Chatzopoulou, N. Wang, F. Gao, I. Al-Ramahi, X.H. Lu, E.M. Ramos, K. El-Zein, Y. Zhao, S. Deverasetty, A. Tebbe, C. Schaab, D.J. Lavery, D. Howland, S. Kwak, J. Botas, J.S. Aaronson, J. Rosinski, G. Coppola, S. Horvath, X.W. Yang, Integrated genomics and proteomics define huntingtin CAG length-dependent networks in mice, Nat. Neurosci. 19 (2016) 623-633.

[4] S. Aronov, G. Aranda, L. Behar, I. Ginzburg, Axonal tau mRNA localization coincides with tau protein in living neuronal cells and depends on axonal targeting signal, J. Neurosci. 21 (2001) 6577-6587.

[5] Y.H. Li, H. Werner, A.W. Puschel, Rheb and mTOR regulate neuronal polarity through Rap1B, J. Biol. Chem. 283 (2008) 33784-33792.

[6] T. Morita, K. Sobue, Specification of neuronal polarity regulated by local translation of CRMP2 and Tau via the mTOR-p70S6K pathway, J. Biol. Chem. 284 (2009) 27734-27745. 
[7] M.L. Caillet-Boudin, L. Buee, N. Sergeant, B. Lefebvre, Regulation of human MAPT gene expression, Mol. Neurodegener. 10 (2015) 28.

[8] J.C. Larcher, L. Gasmi, W. Viranaicken, B. Edde, R. Bernard, I. Ginzburg, P. Denoulet, Ilf3 and NF90 associate with the axonal targeting element of Tau mRNA, FASEB J. 18 (2004) 1761-1763.

[9] R. Atlas, L. Behar, E. Elliott, I. Ginzburg, The insulin-like growth factor mRNA binding-protein IMP-1 and the Ras-regulatory protein G3BP associate with tau mRNA and HuD protein in differentiated P19 neuronal cells, J. Neurochem. 89 (2004) 613-626.

[10] Z. Tang, E. Ioja, E. Bereczki, K. Hultenby, C. Li, Z. Guan, B. Winblad, J.J. Pei, mTor mediates tau localization and secretion: implication for Alzheimer's disease, Biochim. Biophys. Acta 1853 (2015) 1646-1657.

[11] Y.X. Sun, X. Ji, X. Mao, L. Xie, J. Jia, V. Galvan, D.A. Greenberg, K. Jin, Differential activation of mTOR complex 1 signaling in human brain with mild to severe Alzheimer's disease, J. Alzheimers Dis. 38 (2014) 437-444.

[12] J.R. Dickson, C. Kruse, D.R. Montagna, B. Finsen, M.S. Wolfe, Alternative polyadenylation and miR-34 family members regulate tau expression, J. Neurochem. 127 (2013) 739-749.

[13] J.E. Cohen, P.R. Lee, R.D. Fields, Systematic identification of 3'-UTR regulatory elements in activity-dependent mRNA stability in hippocampal neurons, Philos. Trans. R. Soc. Lond. B Biol. Sci. 369 (2014).

[14] P. Lau, K. Bossers, R. Janky, E. Salta, C.S. Frigerio, S. Barbash, R. Rothman, A.S, Sierksma, A. Thathiah, D. Greenberg, A.S. Papadopoulou, T. Achsel, T. Ayoubi, H. Soreq, J. Verhaagen, D.F. Swaab, S. Aerts, B. De Strooper, Alteration of the microRNA network during the progression of Alzheimer's disease, EMBO Mol. Med. 5 (2013) 1613-1634.

[15] I. Santa-Maria, M.E. Alaniz, N. Renwick, C. Cela, T.A. Fulga, D. Van Vactor, T. Tuschl, L.N. Clark, M.L. Shelanski, B.D. McCabe, J.F. Crary, Dysregulation of microRNA-219 promotes neurodegeneration through post-transcriptional regulation of tau, J. Clin. Invest. 125 (2015) 681-686.

[16] N.A. Twine, K. Janitz, M.R. Wilkins, M. Janitz, Whole transcriptome sequencing reveals gene expression and splicing differences in brain regions affected by Alzheimer's disease, PLoS One 6 (2011) e16266.

[17] D. Kim, G. Pertea, C. Trapnell, H. Pimentel, R. Kelley, S.L. Salzberg, TopHat2: accurate alignment of transcriptomes in the presence of insertions, deletions and gene fusions, Genome Biol. 14 (2013) R36.

[18] H. Li, B. Handsaker, A. Wysoker, T. Fennell, J. Ruan, N. Homer, G. Marth, G. Abecasis, R. Durbin, The sequence alignment/map format and SAM tools, Bioinformatics (Oxf. Engl.) 25 (2009) 2078-2079.

[19] M. Novak, R. Jakes, P.C. Edwards, C. Milstein, C.M. Wischik, Difference between the tau protein of Alzheimer paired helical filament core and normal tau revealed by epitope analysis of monoclonal antibodies 423 and 7.51, Proc. Natl. Acad. Sci. U. S. A. 88 (1991) 5837-5841.

[20] M. Hokama, S. Oka, J. Leon, T. Ninomiya, H. Honda, K. Sasaki, T. Iwaki, T. Ohara, T. Sasaki, F.M. LaFerla, Y. Kiyohara, Y. Nakabeppu, Altered expression of diabetes-related genes in Alzheimer's disease brains: the Hisayama study, Cereb. Cortex 24 (2014) 2476-2488

[21] J.L. Zamanian, L. Xu, L.C. Foo, N. Nouri, L. Zhou, R.G. Giffard, B.A. Barres, Genomic analysis of reactive astrogliosis, J. Neurosci. 32 (2012) 6391-6410.

[22] A. Kocabas, T. Duarte, S. Kumar, M.A. Hynes, Widespread differential expression of coding region and 3' UTR sequences in neurons and other tissues, Neuron 88 (2015) 1149-1156.

[23] N. Pomara, D. Deptula, R. Singh, Possible muscarinic supersensitivity in Alzheimer's disease, Prog. Clin. Biol. Res. 317 (1989) 1223-1233.

[24] S. Khatoon, I. Grundke-Iqbal, K. Iqbal, Brain levels of microtubule-associated protein tau are elevated in Alzheimer's disease: a radioimmuno-slot-blot assay for nanograms of the protein, J. Neurochem. 59 (1992) 750-753.

[25] H. Braak, E. Braak, Neuropathological stageing of Alzheimer-related changes, Acta Neuropathol. (Berl.) 82 (1991) 239-259.

[26] N. Maphis, G. Xu, O.N. Kokiko-Cochran, A.E. Cardona, R.M. Ransohoff, B.T. Lamb, K. Bhaskar, Loss of tau rescues inflammation-mediated neurodegeneration, Front. Neurosci. 9 (2015) 196.
[27] C.K. Glass, K. Saijo, B. Winner, M.C. Marchetto, F.H. Gage, Mechanisms underlying inflammation in neurodegeneration, Cell 140 (2010) 918-934.

[28] A.J. Barton, P.J. Harrison, A. Najlerahim, J. Heffernan, B. McDonald, J.R. Robinson, D.C. Davies, W.J. Harrison, P. Mitra, J.A. Hardy, et al., Increased tau messenger RNA in Alzheimer's disease hippocampus, Am. J. Pathol. 137 (1990) 497-502.

[29] K. Le Guennec, O. Quenez, G. Nicolas, D. Wallon, S. Rousseau, A.C. Richard, J. Alexander, P. Paschou, C. Charbonnier, C. Bellenguez, B. Grenier-Boley, D. Lechner, M.T. Bihoreau, R. Olaso, A. Boland, V. Meyer, J.F. Deleuze, P. Amouyel, H.M. Munter, G. Bourque, M. Lathrop, T. Frebourg, R. Redon, L. Letenneur, J.F. Dartigues, O. Martinaud, O. Kalev, S. Mehrabian, L. Traykov, T. Strobel, I. Le Ber, P. Caroppo, S. Epelbaum, T. Jonveaux, F. Pasquier, A. Rollin-Sillaire, E. Genin, L. Guyant-Marechal, G.G. Kovacs, J.C. Lambert, D. Hannequin, D. Campion, A. Rovelet-Lecrux, 17q21.31 duplication causes prominent tau-related dementia with increased MAPT expression, Mol. Psychiatry (2016).

[30] G. Mangin, D. Couchie, C. Charriere-Bertrand, J. Nunez, Timing of expression of tau and its encoding mRNAs in the developing cerebral neocortex and cerebellum of the mouse, J. Neurochem. 53 (1989) 45-50.

[31] D. Couchie, F. Legay, J. Guilleminot, F. Lebargy, J.P. Brion, J. Nunez, Expression of Tau protein and Tau mRNA in the cerebellum during axonal outgrowth, Exp. Brain Res. 82 (1990) 589-596.

[32] J.N. Keller, K.B. Hanni, W.R. Markesbery, Impaired proteasome function in Alzheimer's disease, J. Neurochem. 75 (2000) 436-439.

[33] Y. Wang, E. Mandelkow, Degradation of tau protein by autophagy and proteasomal pathways, Biochem. Soc. Trans. 40 (2012) 644-652.

[34] P. Martin-Maestro, R. Gargini, G. Perry, J. Avila, V. Garcia-Escudero, PARK2 enhancement is able to compensate mitophagy alterations found in sporadic Alzheimer's disease, Hum. Mol. Genet. 25 (2016) 792-806.

[35] A. Wyttenbach, S. Hands, M.A. King, K. Lipkow, A.M. Tolkovsky, Amelioration of protein misfolding disease by rapamycin: translation or autophagy? Autophagy 4 (2008) 542-545.

[36] L. Martin, X. Latypova, F. Terro, Post-translational modifications of tau protein: implications for Alzheimer's disease, Neurochem. Int. 58 (2011) 458-471.

[37] A.G. Hinnebusch, I.P. Ivanov, N. Sonenberg, Translational control by 5'-untranslated regions of eukaryotic mRNAs, Science 352 (2016) 1413-1416.

[38] S. Aronov, R. Marx, I. Ginzburg, Identification of 3'UTR region implicated in tau mRNA stabilization in neuronal cells, J. Mol. Neurosci. 12 (1999) 131-145.

[39] T.R. Mercer, D. Wilhelm, M.E. Dinger, G. Solda, D.J. Korbie, E.A. Glazov, V. Truong, M. Schwenke, C. Simons, K.I. Matthaei, R. Saint, P. Koopman, J.S. Mattick, Expression of distinct RNAs from 3' untranslated regions, Nucleic Acids Res. 39 (2011) 2393-2403.

[40] E.D. Roberson, K. Scearce-Levie, J.J. Palop, F. Yan, I.H. Cheng, T. Wu, H. Gerstein, G.Q. Yu, L. Mucke, Reducing endogenous tau ameliorates amyloid beta-induced deficits in an Alzheimer's disease mouse model, Science 316 (2007) 750-754

[41] B.B. Holmes, M.I. Diamond, Prion-like properties of Tau protein: the importance of extracellular Tau as a therapeutic target, J. Biol. Chem. 289 (2014) 19855-19861.

[42] S.L. DeVos, D.K. Goncharoff, G. Chen, C.S. Kebodeaux, K. Yamada, F.R. Stewart, D.R. Schuler, S.E. Maloney, D.F. Wozniak, F. Rigo, C.F. Bennett, J.R. Cirrito, D.M. Holtzman, T.M. Miller, Antisense reduction of tau in adult mice protects against seizures, J. Neurosci. 33 (2013) 12887-12897.

[43] L.M. Ittner, Y.D. Ke, F. Delerue, M. Bi, A. Gladbach, J. van Eersel, H. Wolfing, B.C. Chieng, M.J. Christie, I.A. Napier, A. Eckert, M. Staufenbiel, E. Hardeman, J. Gotz, Dendritic function of tau mediates amyloid-beta toxicity in Alzheimer's disease mouse models, Cell 142 (2010) 387-397.

[44] Y. Yin, D. Gao, Y. Wang, Z.H. Wang, X. Wang, J. Ye, D. Wu, L. Fang, G. Pi, Y. Yang, X.C. Wang, C. Lu, K. Ye, J.Z. Wang, Tau accumulation induces synaptic impairment and memory deficit by calcineurin-mediated inactivation of nuclear CaMKIV/CREB signaling, Proc. Natl. Acad. Sci. U. S. A. (2016) 